\title{
ARTICLE Oxytocin reduces a chemosensory-induced stress bias in social perception
}

Ayline Maier ${ }^{1}$, Dirk Scheele ${ }^{1}$, Franny B. Spengler ${ }^{1}$, Tugba Menba ${ }^{1}$, Franziska Mohr ${ }^{1}$, Onur Güntürkün ${ }^{2}$, Birgit Stoffel-Wagner ${ }^{3,4}$, Thomas M. Kinfe ${ }^{1}$, Wolfgang Maier ${ }^{4,5}$, Sahib S. Khalsa ${ }^{6,7}$ and René Hurlemann (D) $^{1,4}$

Social transmission of fear is not restricted to visual or auditory cues, but extends to the phylogenetically more ancient olfactory domain. Anxious individuals exhibit heightened sensitivity towards chemosensory stress signals in sweat; however, it is still unknown whether endogenous neuromodulators such as the peptide hormone oxytocin (OXT) influence the chemosensory communication of stress. Here, we investigated whether OXT selectively diminishes behavioral and neural responses to social chemosensory stress cues utilizing a randomized, double-blind, placebo (PLC)-controlled, within-subject functional MRI study design. Axillary sweat was obtained from 30 healthy male donors undergoing the Trier Social Stress Test (stress) and bicycle ergometer training (sport). Subsequently, 58 healthy participants (30 females) completed a forced-choice emotional face recognition task with stimuli of varying intensities (neutral to fearful) while they were exposed to both sweat stimuli and a nonsocial control odor following intranasal OXT or PLC administration, respectively. OXT diminished stress-induced recognition accuracy and response time biases towards fear. On the neural level, OXT reduced stress-evoked responses in the amygdala in both sexes, the anterior cingulate cortex (ACC) in females, and the hippocampus in males. Furthermore, OXT reinstated the functional connectivity between the ACC and the fusiform face area that was disrupted by stress odors under PLC. Our findings reveal a new role for OXT signaling in the modulation of chemosensory communication of stress in humans. Mechanistically, this effect appears to be rooted in a downregulation of stress-induced limbic activations and concomitant strengthening of top-down control descending from the ACC to the fusiform face area.

Neuropsychopharmacology (2019) 44:281-288; https://doi.org/10.1038/s41386-018-0063-3

\section{INTRODUCTION}

Homeostatic threats trigger a well-orchestrated stress cascade of neuroendocrine, autonomic, and behavioral changes [1]. It is well established that persistence of these changes, due to dysregulations of allostatic systems and/or chronic exposure to stressors, plays a key role in the pathophysiology of stress-related psychiatric disorders [2]. From a social neuroscience perspective, stress is highly contagious, i.e., even witnessing others experiencing psychosocial distress can elicit strong vicarious stress responses, evident in heightened cortisol levels [3] and elevated heart rate [4].

Mounting evidence indicates that not only rats [5] but also humans communicate distress and "alarm states" via chemosensory signals. For instance, exposure to sweat from healthy volunteers undergoing an emotional stressor task augments the startle response [6], and induces a bias towards perceiving ambiguous facial expressions as more fearful [7] or threatening [8]. On the neural level, human chemosensory stress signals have been suggested to be processed outside the main olfactory system. As opposed to common odors, they do not recruit the primary olfactory cortex (i.e., the piriform and the entorhinal cortex), but rather a specialized neural network associated with the processing of socioemotional information [9] including the amygdala, hippocampus, and anterior cingulate cortex (ACC) $[8,10,11]$. Both amygdala and ACC activation in response to social odors may reflect threat detection $[12,13]$, while hippocampal activation has been linked to odor recognition memory [14]. Furthermore, women seem to be more receptive to stress-related olfactory cues compared to men, with women exhibiting a reduced positive emotional priming effect [15] and enhanced neural responses in the prefrontal cortex [16]. Importantly, individuals with increased stress vulnerability, such as patients with social anxiety [17] or panic disorder [18], display heightened sensitivity towards these olfactory stress cues. Olfactory dysfunctions have also been described as a feature of the clinical phenotype of other psychiatric disorders including schizophrenia, depression [19], and post-traumatic stress disorder [20], rendering the olfactory pathway a potentially pivotal target for therapeutic interventions.

The hypothalamic peptide hormone oxytocin (OXT) has been identified as a promising therapeutic agent for attenuating maladaptive stress responses [21]. Evidence for anti-stress and

\footnotetext{
${ }^{1}$ Division of Medical Psychology, University of Bonn, Bonn 53105, Germany; ${ }^{2}$ Institute for Cognitive Neuroscience, Biopsychology, Ruhr-University of Bochum, Bochum 44780, Germany; ${ }^{3}$ Institute for Clinical Chemistry and Pharmacology, University of Bonn, Bonn 53105, Germany; ${ }^{4}$ Department of Psychiatry, University of Bonn, Bonn 53105 , Germany;

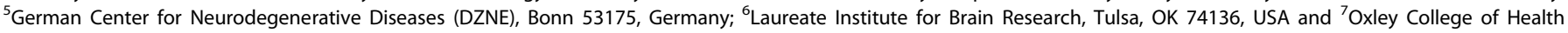
Sciences, University of Tulsa, Tulsa, OK 74104, USA

Correspondence: Dirk Scheele (Dirk-Scheele@gmx.de) or René Hurlemann (renehurlemann@me.com)

These authors contributed equally: Ayline Maier, Dirk Scheele.
}

Received: 14 September 2017 Revised: 2 March 2018 Accepted: 4 April 2018

Published online: 12 April 2018 
A

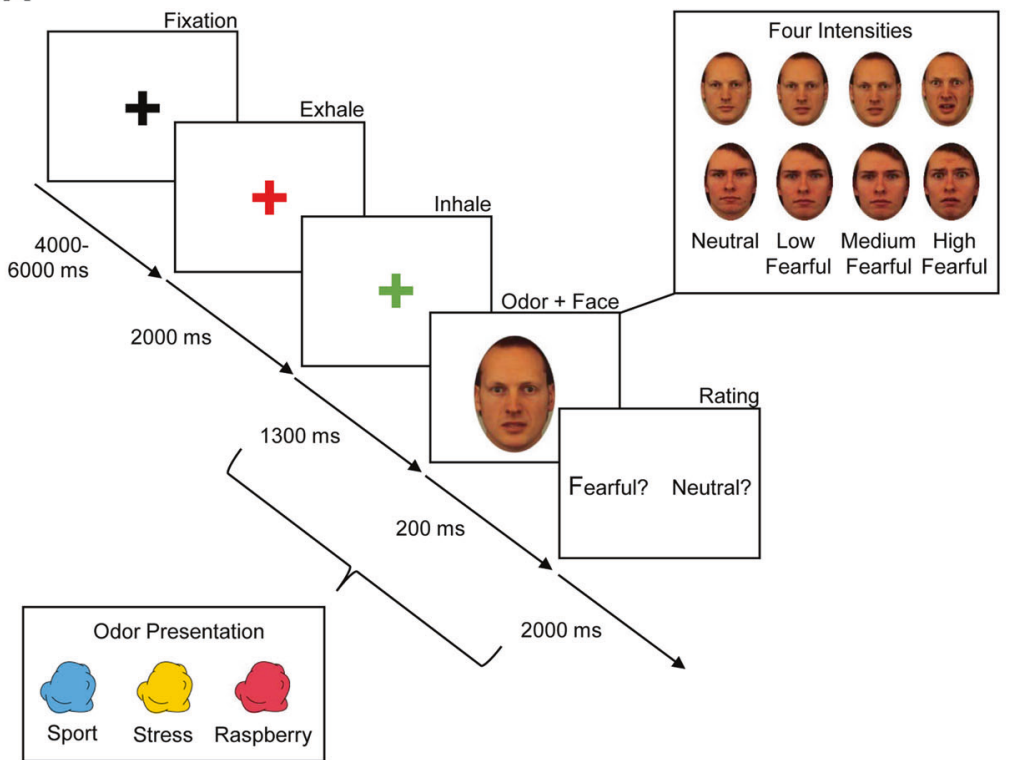

B

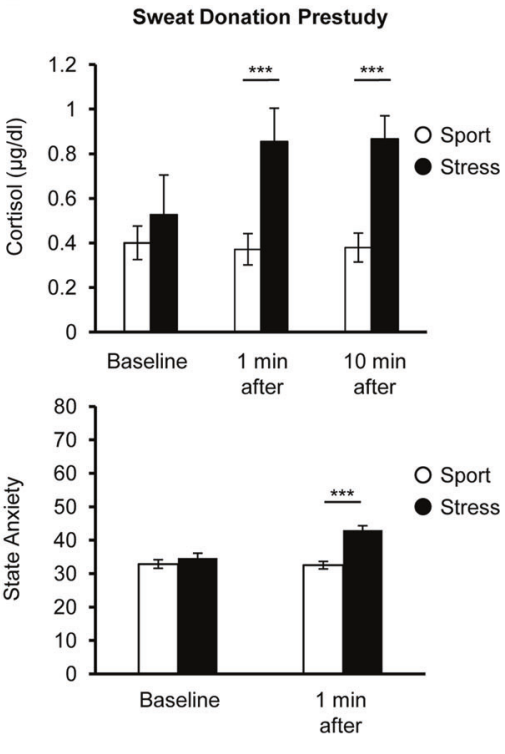

Fig. 1 Task design. Participants were presented with stress sweat, sport sweat, or raspberry odor (as a non-social control condition) during a forced-choice emotion recognition task (a). Odor delivery via olfactometer was synchronized with respiratory cues, with the participants being instructed to breathe orthonasally and inhale on cue throughout the experiment. In each trial, olfactory stimulus delivery spanned the inhalation cue as well as the face stimulus for a total duration of $1500 \mathrm{~ms}$. Trials were separated by a jittered inter-stimulus interval (4-6s; mean: $5 \mathrm{~s}$ ) and the next trial started immediately after the response was recorded or after $2000 \mathrm{~ms}$ if no response was made. Each of the three olfactory stimulus types was presented 48 times in random order, resulting in 144 trials over a period of about 20 min. Physiological and psychological measurements of the sweat donors (b). Salivary cortisol levels and state anxiety ratings of sweat donors were elevated after completing the Trier Social Stress Test (stress condition) compared to bicycle ergometer training (sport condition). Error bars represent SEM. $* * * P<0.001$

anxiolytic effects of OXT accrued from studies showing that in humans intranasally administered OXT enhances the stressbuffering effects of social support [22], reduces amygdala responses to fearful faces [23], and facilitates fear extinction [24]. Surprisingly, little is known about the neuromodulatory role of OXT on olfactory processing in humans. In mice, OXT knock-outs fail to develop olfactory-based social memory [25] despite an intact detection of non-social olfactory stimuli, and impaired social recognition that can be restored by bilateral microinjections of OXT into the amygdala [26]. In patients with schizophrenia, lower OXT plasma levels have been associated with poorer recognition accuracy of pleasant and unpleasant odors [27], whereas a single dose of intranasal OXT improved the olfactory detection thresholds for synthetic fragrance [28]. It is currently unclear whether OXT also modulates chemosensory-induced stress responses in humans.

To address this question, we conducted a randomized, doubleblind, placebo-controlled, within-subject functional magnetic resonance imaging (fMRI) study involving 58 healthy participants. Participants completed a forced-choice emotional face recognition task (cf. Fig. 1a) using stimuli of varying intensities (neutral to fearful), while they were exposed to sweat stimuli and a non-social control odor (raspberry) after intranasal placebo (PLC) and OXT administration, respectively. Social chemosensory stimuli were obtained from axillary sweat of an independent sample of 30 healthy male donors undergoing the Trier Social Stress Test protocol (TSST; stress condition) [29] and ergometer training (sport condition) as a control. Given the anxiolytic and anti-stress profile of OXT, we hypothesized that OXT would selectively diminish stress-induced behavioral biases and neural responses in the amygdala, hippocampus, and ACC to stress-related odors. Additionally, we assumed that OXT would modulate this crossmodal sensory integration by altering the functional connectivity between odor-sensitive regions (i.e., amygdala, hippocampus, and
ACC) and visual processing areas (i.e., fusiform face area (FFA)). Moreover, based on prior findings of differential responses to stress-related chemosignals in women and men, we explored sex as a potential moderator variable across our analyses.

\section{MATERIALS AND METHODS}

\section{Participants}

We tested a total of 60 healthy, right-handed, heterosexual, nonsmoking volunteers (30 females). All participants provided written informed consent. The study was approved by the institutional review board of the Medical Faculty of the University of Bonn and carried out in accordance with the latest revision of the Declaration of Helsinki. Subjects were free of current and past physical or psychiatric illness, as assessed by medical history and the Mini-International Neuropsychiatric Interview (MINI) [30] prior to enrollment in the study. Additionally, all participants were screened for anosmia administering an extended odor test that comprised both an odor identification and discrimination task (Burghart GmbH, Burghart Wedel, Germany). None of the participants were pregnant or used hormonal contraceptives during the study. Two male participants were excluded from the study due to anosmic threshold scores (olfactory threshold $<6$ ), resulting in 58 participants (30 females, mean \pm SD age, $24.90 \pm$ 3.11 years) for the behavioral analyses. Moreover, 8 participants were excluded due to technical malfunctions or excessive head motion $\left(>3 \mathrm{~mm} /{ }^{\circ}\right.$ ) during scanning, leaving 50 participants (26 females, mean $\pm S D$ age, $24.54 \pm 3.09$ years) for the fMRI data analyses.

\section{Olfactory stimuli and delivery}

Subjects were exposed to three different odor stimuli during the fMRI experiment: (i) stress sweat and (ii) sport sweat, both of which were obtained from an independent sample of 30 healthy male 
A

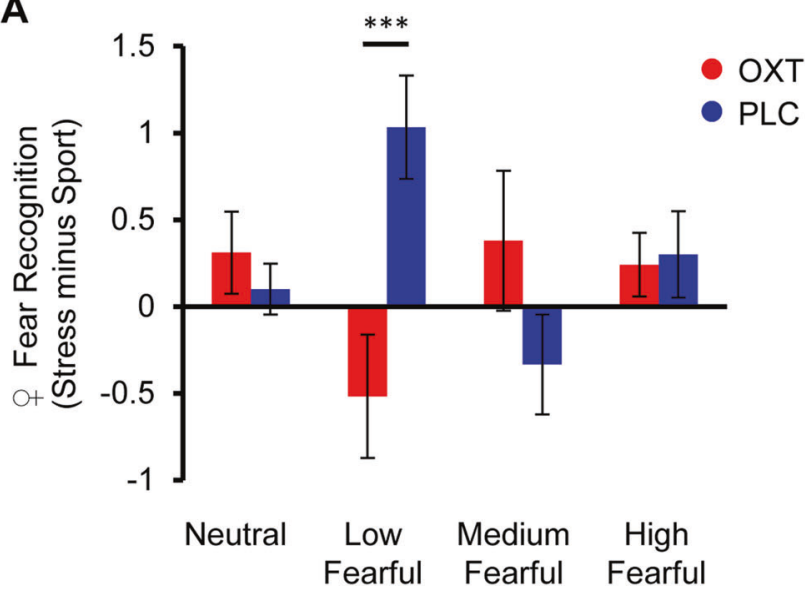

B

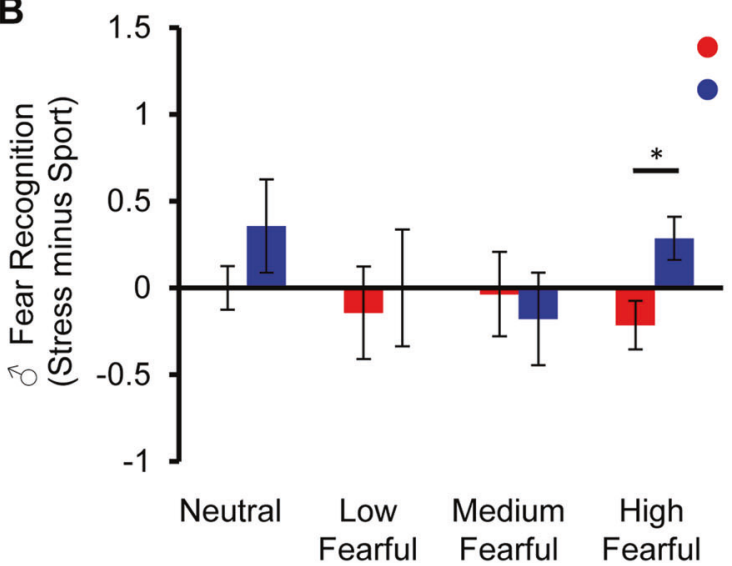

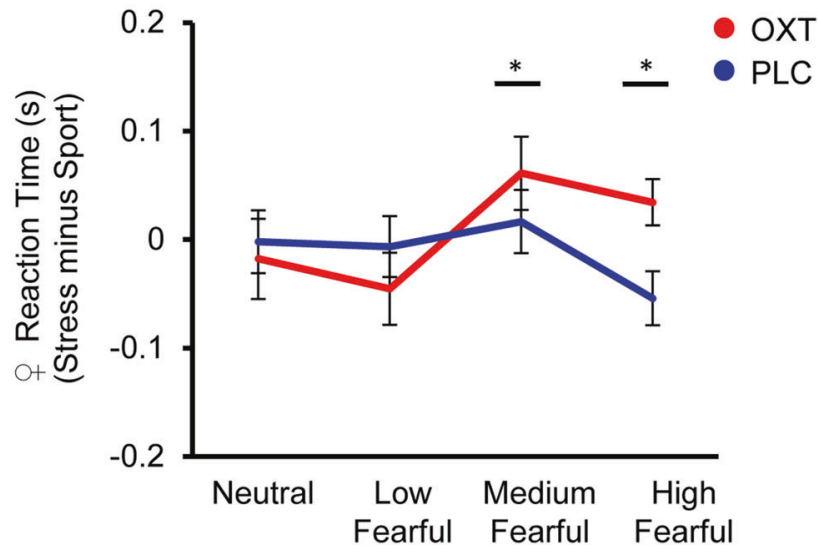

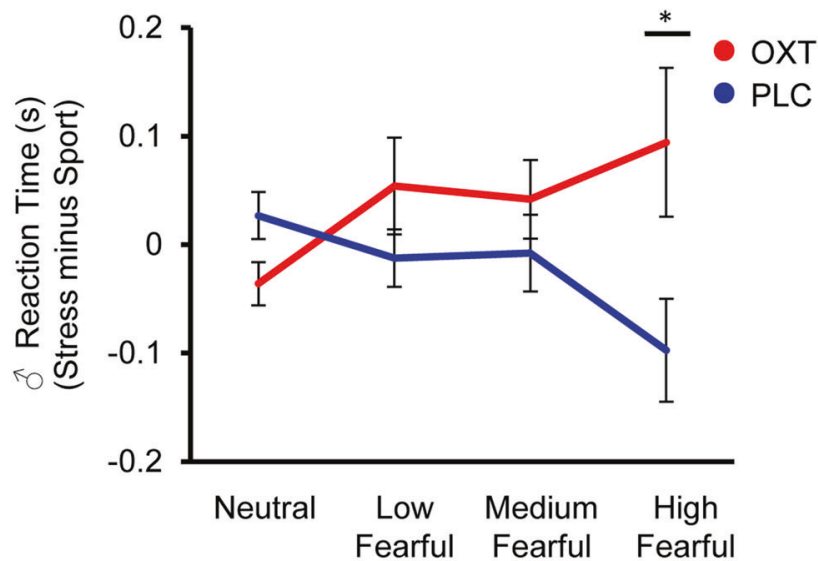

Fig. 2 Effects of oxytocin (OXT) on odor-induced biases in emotion recognition and response times in women (a) and men (b). Stress sweat biased women to perceive ambiguous faces with a low fearful facial expression as more fearful and to rate medium and high fearful faces more quickly. Stress sweat biased men to recognize more faces as fearful in the high fearful condition and rate high fearful faces more quickly. These biases vanished under OXT. Error bars represent SEM. OXT oxytocin, PLC placebo. ${ }^{* * * P<0.001 ;{ }^{*} P<0.05}$

donors (mean \pm SD age, $23.30 \pm 2.67$ years) who underwent the TSST [29] and an ergometer training as a control (cf. Supplementary Information (SI)), and (iii) chemically synthesized raspberry (Burghart $\mathrm{GmbH}$, Wedel, Germany) was used as a non-social control odor. Sweat donors experienced significantly greater psychological stress during the stress condition compared to the sport condition, which was reflected in elevated salivary cortisol levels and state anxiety ratings (cf. Fig. $1 \mathrm{~b}$, cf. SI).

Olfactory stimuli were presented using a three-channel computer-controlled MRI compatible air-dilution olfactometer (OG001, Burghart GmbH, Wedel, Germany). Respiratory compliance was monitored online throughout the fMRI paradigm using an MR-compatible breathing belt (RX-TSD221-MRI) affixed to the subject's chest to ensure that inhalations occurred during odor delivery.

\section{Odor stimuli rating}

To examine a possible modulatory effect of OXT on odor assessment, participants rated the pleasantness, intensity, and familiarity of the three olfactory stimuli used in the fMRI task at the end of each testing session. (cf. SI). In addition, we conducted a complementary study to evaluate whether sport and stress sweat stimuli were comparable across all scan sessions. An unrelated sample of 29 healthy participants (18 females, mean \pm SD age, $24.93 \pm 3.62$ years) rated the pleasantness, intensity, and familiarity of all sweat odor stimuli.
Experimental design and procedures

We performed a randomized, double-blind, PLC-controlled, crossover design study. The two fMRI testing sessions were separated by a minimum of $24 \mathrm{~h}$. In both testing sessions, participants selfadministered $40 \mathrm{IU}$ synthetic OXT (Novartis, Basel, Switzerland) or PLC via nasal spray under the supervision of an experimenter. The dose of $40 \mathrm{IU}$ OXT has previously been demonstrated to alter the olfactory perception in patients with schizophrenia [28]. The PLC solution contained identical ingredients except for the peptide itself (cf. SI). Functional MRI scanning started 30 min after nasal administration. Moreover, the participants' olfactory threshold was verified following nasal spray administration in both sessions (cf. $\mathrm{SI})$.

fMRI paradigm

For the fMRI scan, we employed a modified version of an established facial fear recognition task [8]. Utilizing a forced-choice paradigm, participants were instructed to decide whether face stimuli presented at varying salience (neutral, fearful ranging from low to high intensity) display a neutral or fearful expression while they were exposed to the three olfactory stimuli (cf. Fig. 1a, cf. SI).

fMRI data acquisition and analysis

Data were collected on a $3 \mathrm{~T}$ MRI system (Siemens, Erlangen, Germany) using gradient-echo echo-planar imaging. FMRI data were preprocessed and analyzed using SPM12 software 

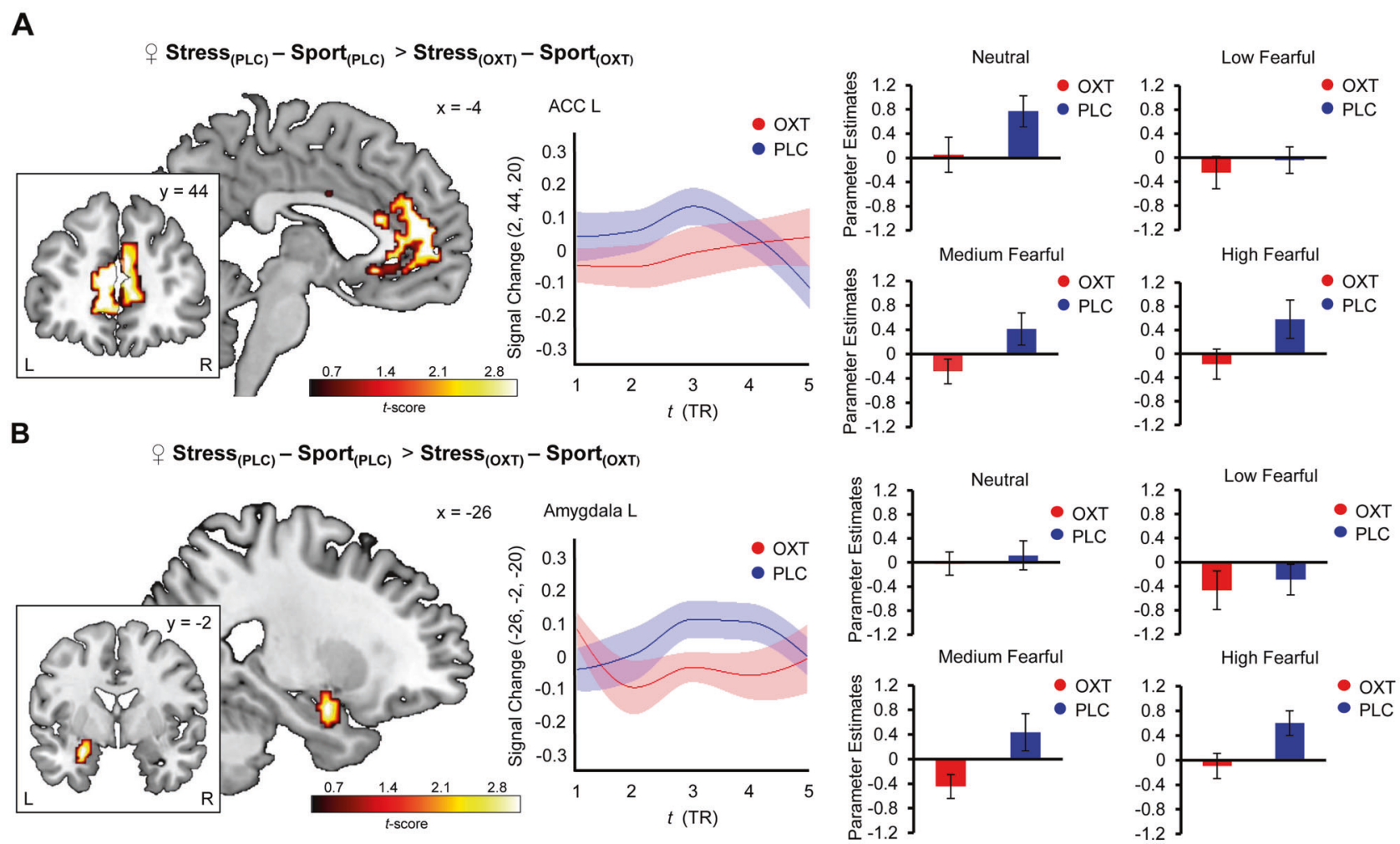

Fig. 3 Effects of oxytocin (OXT) on stress-specific neural responses in women. OXT reduced stress-specific activations in the bilateral anterior cingulate cortex (ACC; $\mathbf{a}$ ) and in the left amygdala (b) across all emotion intensity levels. Error bars and the shaded area indicate the SEM. ACC anterior cingulate, L left hemisphere, OXT oxytocin, PLC placebo, R right hemisphere

(Wellcome Trust Centre for Neuroimaging, London, UK; http:// www.fil.ion.ucl.ac.uk/spm) implemented in Matlab R2010b (The MathWorks Inc., Natick, MA). Results were assessed using a GLM (general linear model) approach (for details cf. SI).

Connectivity analysis

To address the neuromodulatory effects of OXT on functional connectivity in response to chemosensory cues, we performed a generalized form of context-dependent psychophysiological interaction interactions analysis (gPPl; http://www.nitrc.org/ projects/gppi) [31]. Seed regions were identified as $4 \mathrm{~mm}$ radius spheres centered at the maximum $t$-value of significant clusters obtained in BOLD (blood oxygen dependent level) analysis.

\section{RESULTS}

Fear recognition and response latency

Analyses of variance (ANOVAs) yielded a higher-order interaction effect of sex (cf. SI) on fear recognition and a main effect on response time (RT), indicating that female and male participants responded differently to chemosensory cues. Thus, we proceeded all analyses separated by sex and conducted two ANOVAs with the within-subject factors treatment (PLC, OXT), sweat type (stress, sport), and emotion intensity (neutral, low, medium, and high fearful).

\section{Female participants}

For women, we observed treatment $\times$ sweat typexintensity interaction effects on fear recognition $\left(F_{(2.44,68.31)}=6.96, P=0.001, \eta_{\mathrm{p}}{ }^{2}\right.$ $=0.2)$ and $\operatorname{RT}\left(F_{(3,81)}=4.71, P=0.004, \eta_{p}{ }^{2}=0.15\right)$. Using fear recognition ratings as dependent variable, we found a sweat typexintensity interaction $\left(F_{(3,87)}=5.98, P=0.001, \eta_{p}{ }^{2}=0.17\right)$ under PLC. Post hoc $t$-tests confirmed that under PLC, stress sweat compared to sport sweat biased females to perceive ambiguous faces (i.e., low fearful expression) as more fearful $\left(t_{(29)}\right.$ $=3.67, P=0.001, d=0.34$ ) (cf. Fig. 2a). Using RT as dependent variable, the analysis revealed a trend-to-significant sweat typexintensity interaction $\left(F_{(3,84)}=2.36, P=0.078, \eta_{\mathrm{p}}{ }^{2}=0.08\right)$ in the PLC condition. Exploratory $t$-tests demonstrated that under PLC females responded faster to medium $\left(t_{(28)}=2.2, P=0.036, d=\right.$ $0.28)$ and high fearful $\left(t_{(28)}=2.29, P=0.03, d=0.38\right)$ faces when they were simultaneously confronted with stress sweat compared to sport sweat (cf. Fig. 2a). By contrast, behavioral biases in fear recognition and RT vanished after OXT administration (all PS > 0.05).

Male participants

For men, we found a treatment $\times$ sweat typexintensity interaction effect on RT $\left(F_{(3,81)}=3.28, P=0.025, \eta_{p}{ }^{2}=0.11\right)$, but not on fear recognition. Using fear recognition under PLC as dependent variable, the analysis yielded a main effect of intensity $\left(F_{(1.36,36.73)}\right.$ $\left.=314.82, \quad P<0.001, \eta_{p}{ }^{2}=0.90\right)$, but no intensity $x$ sweat type interaction $(P>0.05)$. Given our a priori hypotheses, we performed exploratory $t$-tests contrasting fear recognition ratings between both sweat type stimuli for all intensities. Results showed that under PLC, males perceived more faces as fearful in the high fearful condition during stress sweat presentation compared to sport sweat presentation $\left(t_{(27)}=2.3, P=0.03, d=0.56\right.$, cf. Fig. $2 b$ ). Likewise, using RT under PLC as dependent variable, exploratory $t$ tests demonstrated that males responded faster to highly salient fearful faces $\left(t_{(27)}=2.08, P=0.049, d=0.56\right)$, during the exposure to stress sweat compared to sport sweat. However, these behavioral biases in fear recognition and RT were absent under OXT (all Ps > 0.05, for more detail, see Sl, cf. Fig. 2b). Together, our findings demonstrate that OXT reversed stress-specific behavioral biases in fear recognition and RT across all participants. 
A

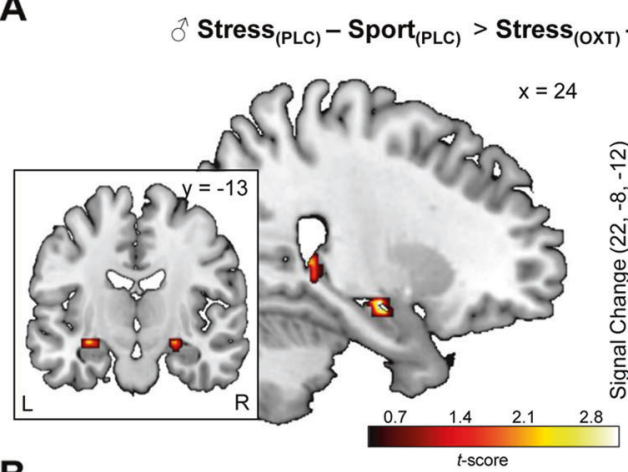

B
${ }_{\text {Stress }}$ PLC) - Sport $_{(\mathrm{PLC})}>$ Stress $_{(\mathrm{OXT})}-$ Sport $_{(\mathrm{OXT})}$

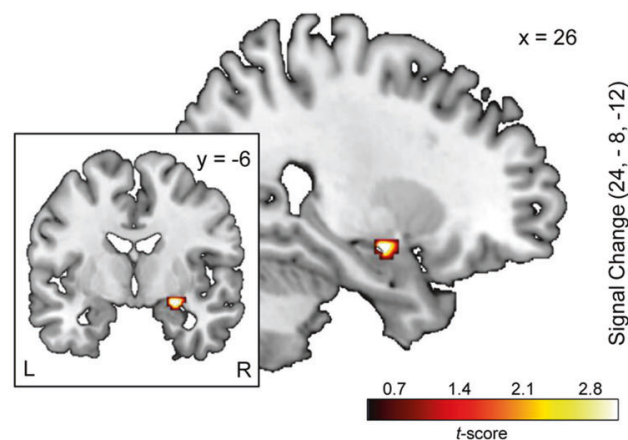

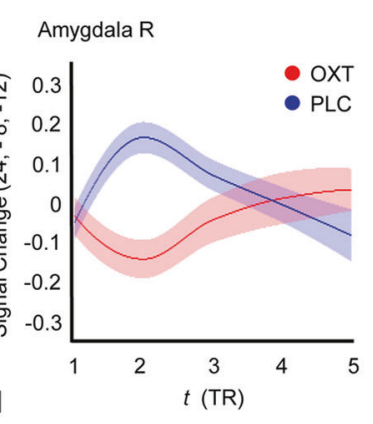
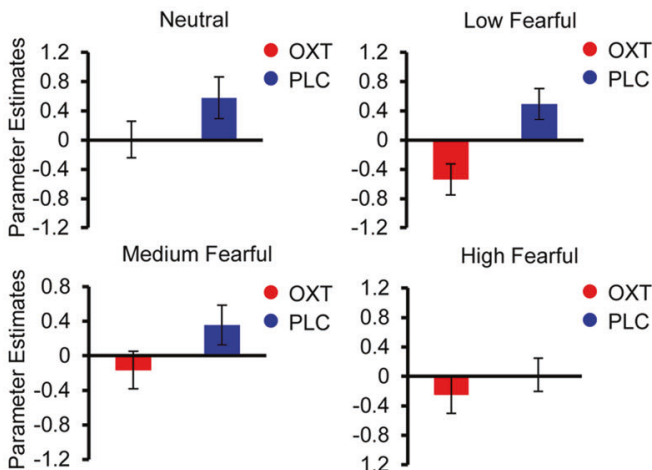

Neutral

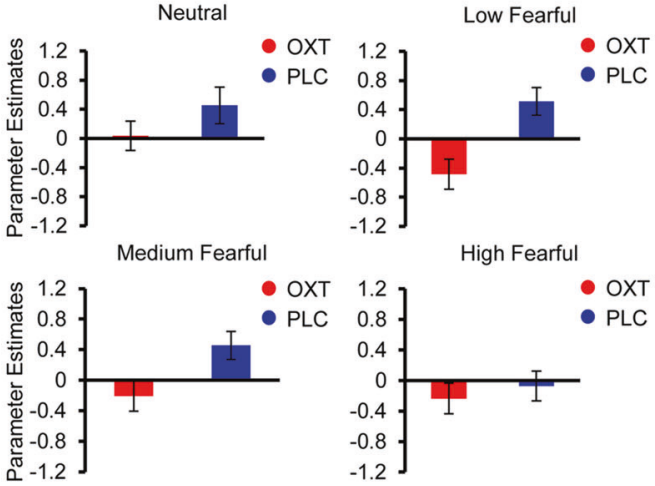

Fig. 4 Effects of oxytocin (OXT) on stress-specific neural responses in men. OXT specifically diminished neural responses to stress sweat in the bilateral hippocampus (a) and in the right amygdala (b) across all emotion intensity levels. Error bars and the shaded area indicate the SEM. $\mathrm{L}$ left hemisphere, OXT oxytocin, PLC placebo, R right hemisphere

fMRI results

Combined group. Due to the absence of intensity-specific effects of chemosensory stress signals $\left[\left(\right.\right.$ Stress $_{(\mathrm{PLC})}-$ Sport $\left.\left._{(\mathrm{PLC})}\right)\right]$ across all participants and within each sex under PLC, stress-induced responses as well as the neuromodulatory effect of OXT on these responses were averaged across all intensity levels. We found that under PLC stress relative to sport sweat elicited increased activation in the right amygdala (peak MNI coordinates $x, y, z$ : $\left.24,-8,-12 ; t_{(294)}=3.72, P_{\mathrm{FWE}}=0.007\right)$ and right ACC $(6,48,8$; $\left.t_{(294)}=3.73, P_{\mathrm{FWE}}=0.025 ; 12,42,2 ; t_{(294)}=3.55, P_{\mathrm{FWE}}=0.049\right)$; additionally, there was a trend-to-significant activation in the right hippocampus $\left(22,-8,-12 ; t_{(294)}=3.44, P_{\mathrm{FWE}}=0.056\right)$. Intranasal OXT administration attenuated stress-associated neural responses in the right amygdala $\left(22,-6,-12 ; t_{(294)}=4.44, P_{\mathrm{FWE}}=0.001\right)$, right hippocampus $\left(22,-8,-12 ; t_{(294)}=3.99, P_{\mathrm{FWE}}=0.01\right)$, and right ACC (8, 24, 16; $\left.t_{(294)}=3.58, P_{\mathrm{FWE}}=0.045\right)$. Moreover, we observed trend-to-significant reductions in stress-specific responses in the left ACC $\left(-8,32,-6 ; t_{(294)}=3.43, P_{\mathrm{FWE}}=0.078\right)$ and left amygdala $\left(-24,0,-18 ; t_{(294)}=2.72, P_{\mathrm{FWE}}=0.099\right)$. OXT did not alter neural responses in the FFA and had no effect on the processing of the non-social control odor (cf. SI).

Female participants. In females, stress relative to sport odor induced responses in the bilateral ACC $\left(2,46,20 ; t_{(150)}=4.27, P_{\mathrm{FWE}}\right.$ $\left.=0.012 ; 6,48,8 ; t_{(150)}=4.00, P_{\mathrm{FWE}}=0.03\right)$ and a trend-tosignificant activation in the left amygdala $\left(-28,0,-22 ; t_{(150)}=\right.$ 3.00, $\left.P_{\mathrm{FWE}}=0.058\right)$ under PLC. Importantly, OXT blunted stressassociated activations in the bilateral ACC $\left(2,46,20 ; t_{(150)}=4.31\right.$, $P_{\mathrm{FWE}}=0.006 ; 2,44,20 ; t_{(150)}=4.35, P_{\mathrm{FWE}}=0.005$, cf. Fig. 3a), left amygdala $\left(-26,-2,-20 ; t_{(150)}=3.21, P_{\mathrm{FWE}}=0.034\right.$, cf. Fig. $\left.3 \mathrm{~b}\right)$, and left hippocampus $\left(-18,-20,-18 ; t_{(150)}=3.69, P_{\mathrm{FWE}}=0.032\right)$.

Male participants. Under PLC, stress compared to sport sweat yielded greater responses in the right amygdala $(24,-8,-14$; $\left.t_{(138)}=3.87, P_{\mathrm{FWE}}=0.006\right)$ and a trend-to-significant increase in activation in the right hippocampus $\left(24,-10,-14 ; t_{(138)}=3.53\right.$, $\left.P_{\mathrm{FWE}}=0.057\right)$ in males. After OXT administration, diminished neural responses to stress sweat were observed in the bilateral hippocampus $\left(22,-8,-12 ; t_{(138)}=3.94, P_{\mathrm{FWE}}=0.016 ;-30,-16\right.$, $-12 ; t_{(138)}=3.96, P_{\mathrm{FWE}}=0.015$, cf. Fig. 4 a) and right amygdala (24, $-8, \quad-12 ; \quad t_{(138)}=4.36, \quad P_{\mathrm{FWE}}=0.001$, cf. Fig. 4b). Extracted parameter estimates confirmed decreased neural responses to stress odor after OXT administration, an effect occurring across intensity levels across all participants. Collectively, our findings demonstrate that intranasal OXT diminishes stress-specific responses.

\section{Connectivity results}

For the right ACC $(6,48,8)$ seed region, gPPI analysis revealed a stress-specific decrease in functional coupling with the left FFA (peak MNI $x, y, z=-24,-78,-10 ; t_{(196)}=4.38, P_{\mathrm{FWE}}=0.004$ ) under PLC across the entire sample. Intriguingly, a whole-brain analysis showed that OXT enhanced functional coupling of the right $A C C(6,48,8)$ to a large-sized cluster extending from the left FFA $\left(-24,-76,-8 ; t_{(196)}=5.27, k_{\mathrm{E}}=23,180, P_{\mathrm{FWE}}<0.01\right)$ to the bilateral lingual gyrus $\left(-24,-52,-8 ; t_{(196)}=5.09 ; 6,-58,4 ; t_{(196)}\right.$ $=4.93$ ) in response to stress sweat relative to sport sweat. Additionally, a region of interest (ROI)-based analysis for the same contrast revealed increased functional connectivity between the right ACC $(6,48,8)$ and the right FFA $\left(28,-60,-2 ; t_{(196)}=4.05\right.$, $\left.P_{\text {FWE }}=0.021\right)$. There were no connectivity changes with the amygdala and hippocampus as seed regions (cf. SI).

Female participants. For females, we found an OXT-induced increase in functional coupling of the left $\operatorname{ACC}(2,44,20)$ and the left FFA $\left(-24,-80,-6 ; t_{(100)}=4.02, P_{\mathrm{FWE}}=0.037\right)$ in response to stress sweat compared to sport sweat (cf. Fig. 5a). Interestingly, a stronger OXT effect on the stress-specific functional coupling of 

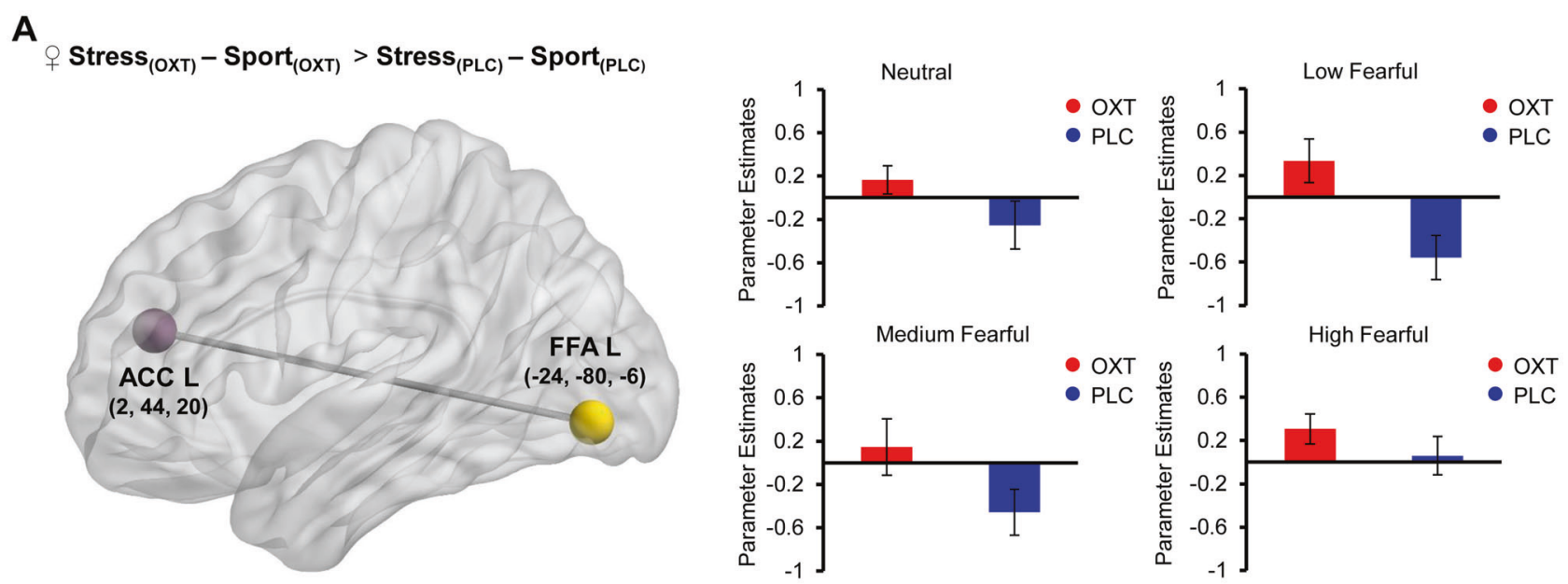

\section{B}
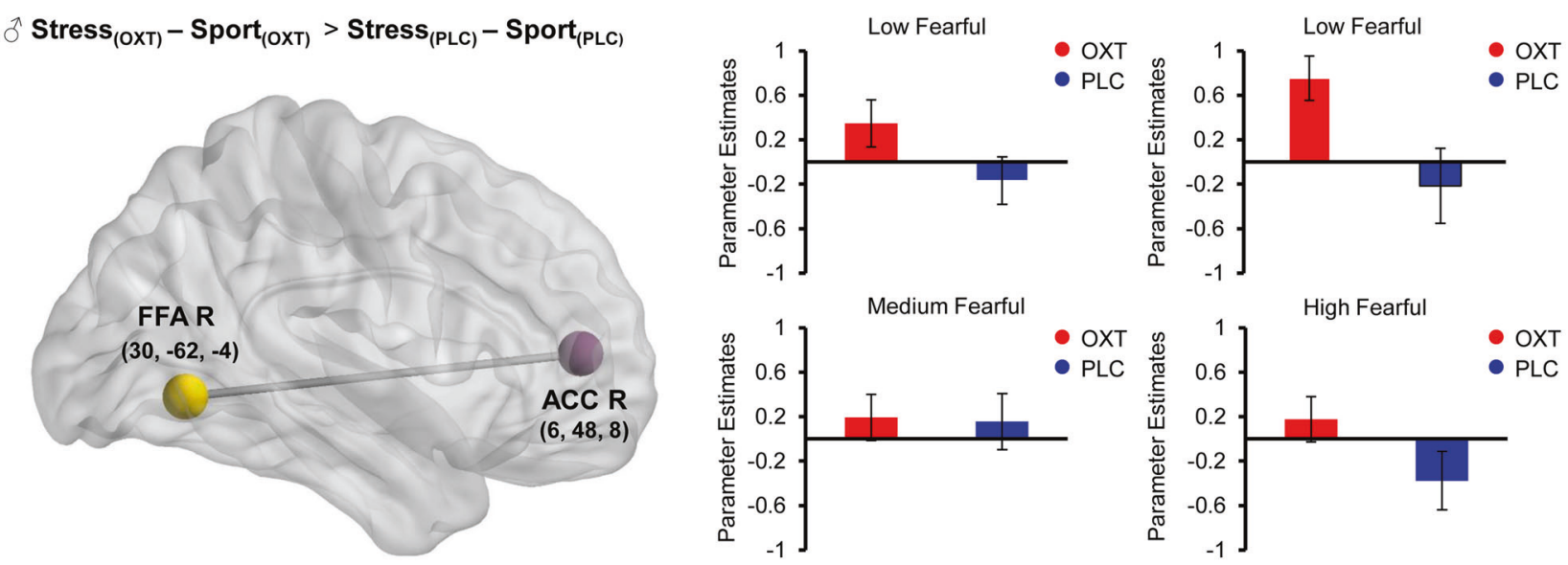

Fig. 5 Effects of oxytocin (OXT) on stress-specific functional connectivity. In women, OXT specifically enhanced functional coupling of the left anterior cingulate cortex (ACC) and the left fusiform face area (FFA) during stress sweat exposure (a). In men, OXT specifically increased functional coupling of the right ACC and the bilateral FFA during stress sweat presentation (b). Identified seed regions of the functional connectivity analysis are depicted as purple spheres and target regions are illustrated as yellow spheres. Error bars represent SEM. ACC anterior cingulate, FFA fusiform face area, L left hemisphere, OXT oxytocin, PLC placebo, R right hemisphere

the left ACC and the right FFA was predictive of a stronger OXT effect on RT ([(Sport ${ }_{(\mathrm{PLC})}>$ Stress $\left._{(\mathrm{PLC})}\right)-\left(\right.$ Sport $_{(\mathrm{OXT})}>$ Stress $\left.\left._{(\mathrm{OXT})}\right)\right] ; r_{(23)}$ $=0.41, P=0.04)$.

Male participants. Whole-brain analyses revealed an OXTfacilitated increase in connectivity of the right ACC $(12,42,2)$ and the left middle frontal gyrus $\left(-28,24,48 ; t_{(92)}=5.32, k_{\mathrm{E}}=127\right.$, $\left.P_{\mathrm{FWE}}=0.017\right)$ in response to stress sweat compared to sport sweat. Moreover, ROI analyses revealed that OXT increased functional coupling of the right ACC $(12,42,2 ; 6,48,8)$ and bilateral FFA $\left(30,-62,-4 ; t_{(92)}=4.15, P_{\mathrm{FWE}}=0.023 ;-26,-58\right.$, $-10 ; t_{(92)}=4.87, P_{\mathrm{FWE}}=0.001$, cf. Fig. $\left.5 \mathrm{~b}\right)$ under stress sweat exposure relative to sport sweat exposure. These results suggest that the perceptual bias induced by chemosensory stress cues may be related to a decreased functional coupling of the ACC involved in the processing of olfactory stress cues and regions such as the FFA encoding fearful facial information. Extracted parameter estimates corroborated increased functional connectivity between ACC seeds and FFA target regions in both sexes. Thus, OXT appeared to reinstate the altered connectivity between olfactory and visual areas, which was paralleled by an absent fear recognition bias.

Odor stimuli ratings

Participants did not rate social chemosensory stimuli differently in the OXT and PLC session with regard to pleasantness (all Ps $>0.1$ ), intensity (all $P \mathrm{~s}>0.5$ ), or familiarity (all $P \mathrm{~s}>0.07$, cf. Supplemental Fig. S2). Thus, significant effects on fear recognition, RT, and neural response patterns cannot be attributed to OXT modulation of odor assessment. Furthermore, the complementary study revealed that social chemosensory stimuli did not exhibit detectable differences in odor quality across all scan sessions (all $P \mathrm{~s}>0.05$, cf. $\mathrm{SI}$ ). This rules out simple odor discrimination as an explanation for the behavioral and neural findings.

\section{DISCUSSION}

In the present study, we sought to determine (i) whether intranasally administered OXT modulates the processing of stress-associated chemosignals and (ii) which neural substrates are involved. Our study utilized a cross-modal paradigm combining the presentation of social odors and fear recognition in faces with varying fear intensities. As expected, our analyses revealed that OXT selectively diminished chemosensory-induced behavioral biases in fear perception and neural responses to stress-associated odors in the amygdala, hippocampus, and ACC. In line with our second hypothesis, connectivity analyses delineated a reinstated functional coupling between the stress-sensitive ACC and the FFA after OXT treatment, highlighting an intensified interaction between olfactory and visual networks.

Our findings closely corroborate previous research by replicating increased stress-specific neural responses in the amygdala [8], 
hippocampus [11], and ACC [10] in humans. The amygdala is a key node in emotion-processing and stress regulation that has been associated with autonomic control [1] and threat detection [12]. Thus, stress-related odors may be evaluated as a potential threat prompting the amygdala to initiate facilitated fear detection in the visual domain. The ACC has been implicated in regulation of behavioral and autonomic responses to emotional and stressful stimuli [32] and executive control in humans [33]. There is evidence that the ACC has a modulatory role in emotion regulation mediated by cingulo-amygdalar connectivity [34]. Increased co-activation with the amygdala may reflect an attentional shift towards threat-relevant cues in fearful faces driven by stress-associated chemosignals. The hippocampus is thought to contribute to threat evaluation through memory retrieval of past experiences [35]. However, it has also been linked to sensory integration of visual and olfactory information [36]. As such, the hippocampus may mediate a cross-modal synthesis of stress-associated chemosignals and fearful faces. These neural activations were paralleled by a stress-induced recognition bias and decreased RT towards fearful faces, suggesting an overall attentional shift towards threatening stimuli. This interpretation is consistent with the documented link of stress-associated chemosignals and heightened vigilance [6].

Interestingly, our results underscore the idea of sex-differential stress responses in encoding negatively valenced stimuli. In accordance with our results, previous studies also reported stronger ACC responses to psychological stress [37] and greater activations in the left amygdala and left ACC in women [38], and enhanced activation in the right amygdala in men [39]. An absent stress-related hippocampus activation may be attributed to neuroprotective effects of heightened estradiol levels [40]. For behavioral responses, we replicated findings that chemosensory stress signals induce a perceptual bias of ambiguous faces in women $[7,8]$. In men, however, we detected a perceptual bias towards high-intensity fearful faces. This is in line with a previous review by Kret and De Gelder [41] showing that women are generally better in recognizing emotions and men exhibit greater responses to threatening cues.

Importantly, we observed that OXT diminished behavioral and neural responses to subtle olfactory social threat signals. These results support and extend prior findings of stress-reducing and anxiolytic effects of OXT during the exposure to a psychosocial stressor [42] and suggest a crucial role of OXT as a potent regulatory system protecting the "social brain" against infiltration from a wealth of incoming subliminal exteroceptive information. The amygdala, hippocampus, and ACC have been identified as OXT binding sites in humans [43] and OXT attenuates threatassociated activations in these regions [23, 44]. Furthermore, the ACC and FFA have been found to be functionally interconnected regions engaged in a cortical top-down face processing network [45] and we observed a positive correlation between OXT-induced behavioral effects and connectivity changes in women. Mechanistically, we suggest that the cross-modal anti-stress effect of OXT may be ascribed to a downregulation of stress-induced amygdala responses and concomitant strengthening of top-down control descending from the ACC to the FFA. These dynamic changes may constitute the underlying substrates mediating the protective effects of OXT in affective face perception. Interestingly, OXT diminished stress-associated behavioral and neural responses to social chemosignals in a sex-independent manner. As expected, OXT did not alter the processing of the non-social odor on the behavioral or neural level (cf. SI).

Our findings raise the question of whether oxytocinergic signaling may constitute an endogenous protection against noxious olfactory-based transmission of fear. Clearly, the neuromodulatory effects of OXT are not restricted to visual and auditory stimuli but also extend to other sensory modalities including effects on interpersonal touch [46] and olfactory signals.
Furthermore, an important translational implication arising from our findings is that individuals with anxiety disorders who exhibit deficient recruitment of top-down control [47] and increased stimulus-driven attention [48] may benefit from OXT treatment. Given the strong context dependency of OXT effects [49], future research is needed to delineate the pathophysiology of this mechanism in the context of stress-related chemosignals and to investigate whether interventions with OXT normalize a pathological hypersensitivity towards stress-related chemosignals in anxiety disorders.

The present study has some limitations. First, the exclusive use of chemosensory cues from male axillary sweat might have promoted the observed sex-specific differences in neural and behavioral response patterns. There is evidence pointing to a distinct chromatographic profile of axillary sweat in men and women [50] implying differential processing of social chemosignals in same-sex and opposite-sex receivers [51]. However, a recent report determined that male axillary sweat produced similar response patterns in male and female participants [52]. Second, in contrast to the intensity-specific behavioral results we did not detect a significant interaction of intensity and odors on the neural level. Future studies should incorporate a more nuanced array of emotion intensities and increase the number of intensity-related trials to improve the power for disentangling intensity-specific neural effects of OXT on chemosensory processing.

Collectively, our data provide first evidence that OXT modulates social threat cues at the olfactory level and exerts a cross-modal anti-stress effect in the visual domain. These findings provide preliminary support for investigating whether endocrinologic interventions with OXT might normalize a pathological chemosensory stress-induced hypersensitivity and perceptual distortions in anxious individuals.

\section{ACKNOWLEDGEMENTS}

The authors wish to thank Paul Jung for programming assistance. The present work was supported by the German Research Foundation (Deutsche Forschungsgemeinschaft (DFG)) Grant (HU1202/4-1 and BE 5465/2-1 to RH and DS).

\section{AUTHOR CONTRIBUTIONS}

AM, DS, and RH designed the experiments; AM, DS, TM, and FM conducted the experiments; AM, DS, FBS, FM, and RH analyzed the data; all authors contributed to writing the paper.

\section{ADDITIONAL INFORMATION}

The online version of this article (https://doi.org/10.1038/s41386-018-0063-3) contains supplementary material, which is available to authorized users.

Competing interests: The authors declare no competing interests.

Publisher's note: Springer Nature remains neutral with regard to jurisdictional claims in published maps and institutional affiliations.

\section{REFERENCES}

1. Ulrich-Lai YM, Herman JP. Neural regulation of endocrine and autonomic stress responses. Nat Rev Neurosci. 2009;10:397-409.

2. McEwen BS, Bowles NP, Gray JD, Hill MN, Hunter RG, Karatsoreos IN, et al. Mechanisms of stress in the brain. Nat Neurosci. 2015;18:1353-63.

3. Engert V, Plessow F, Miller R, Kirschbaum C, Singer T. Cortisol increase in empathic stress is modulated by emotional closeness and observation modality. Psychoneuroendocrinology. 2014;45:192-201.

4. Dimitroff SJ, Kardan O, Necka EA, Decety J, Berman MG, Norman GJ. Physiological dynamics of stress contagion. Sci Rep. 2017;7:6168.

5. Kikusui T, Takigami S, Takeuchi Y, Mori Y. Alarm pheromone enhances stressinduced hyperthermia in rats. Physiol Behav. 2001;72:45-50.

6. Prehn A, Ohrt A, Sojka B, Ferstl R, Pause BM. Chemosensory anxiety signals augment the startle reflex in humans. Neurosci Lett. 2006;394:127-30. 
7. Zhou W, Chen D. Fear-related chemosignals modulate recognition of fear in ambiguous facial expressions. Psychol Sci. 2009;20:177-83.

8. Mujica-Parodi LR, Strey HH, Frederick B, Savoy R, Cox D, Botanov Y, et al. Chemosensory cues to conspecific emotional stress activate amygdala in humans. PLoS ONE. 2009;4:e6415.

9. Lundström JN, Olsson MJ. Functional neuronal processing of human body odors. Vitam Horm. 2010;83:1-23.

10. Prehn-Kristensen A, Wiesner C, Bergmann TO, Wolff $S$, Jansen O, Mehdorn HM, et al. Induction of empathy by the smell of anxiety. PLoS ONE. 2009;4:e5987.

11. Wudarczyk OA, Kohn N, Bergs R, Goerlich KS, Gur RE, Turetsky B, et al. Chemosensory anxiety cues enhance the perception of fearful faces-an fMRI study. Neuroimage. 2016;143:214-22.

12. Adolphs R. Fear, faces, and the human amygdala. Curr Opin Neurobiol. 2008;18:166-72.

13. Mutic S, Brunner YF, Rodriguez-Raecke R, Wiesmann M, Freiherr J. Chemosensory danger detection in the human brain: Body odor communicating aggression modulates limbic system activation. Neuropsychologia. 2017:99:187-98.

14. Lehn $H$, Kjonigsen LJ, Kjelvik G, Haberg AK. Hippocampal involvement in retrieval of odor vs. object memories. Hippocampus. 2013;23:122-8.

15. Pause BM, Ohrt A, Prehn A, Ferstl R. Positive emotional priming of facial affect perception in females is diminished by chemosensory anxiety signals. Chem Senses. 2004;29:797-805.

16. Pause BM, Lubke K, Laudien JH, Ferstl R. Intensified neuronal investment in the processing of chemosensory anxiety signals in non-socially anxious and socially anxious individuals. PLoS ONE. 2010;5:e10342.

17. Pause BM, Adolph D, Prehn-Kristensen A, Ferstl R. Startle response potentiation to chemosensory anxiety signals in socially anxious individuals. Int J Psychophysiol. 2009;74:88-92.

18. Wintermann GB, Donix M, Joraschky P, Gerber J, Petrowski K. Altered olfactory processing of stress-related body odors and artificial odors in patients with panic disorder. PLoS ONE. 2013;8:e74655

19. Atanasova B, Graux J, El Hage W, Hommet C, Camus V, Belzung C. Olfaction: a potential cognitive marker of psychiatric disorders. Neurosci Biobehav Rev. 2008;32:1315-25.

20. Dileo JF, Brewer WJ, Hopwood M, Anderson V, Creamer M. Olfactory identification dysfunction, aggression and impulsivity in war veterans with post-traumatic stress disorder. Psychol Med. 2008;38:523-31.

21. Meyer-Lindenberg A, Domes G, Kirsch P, Heinrichs M. Oxytocin and vasopressin in the human brain: social neuropeptides for translational medicine. Nat Rev Neurosci. 2011;12:524-38.

22. Heinrichs M, Baumgartner T, Kirschbaum C, Ehlert U. Social support and oxytocin interact to suppress cortisol and subjective responses to psychosocial stress. Biol Psychiatry. 2003;54:1389-98.

23. Kirsch P, Esslinger C, Chen Q, Mier D, Lis S, Siddhanti S, et al. Oxytocin modulates neural circuitry for social cognition and fear in humans. J Neurosci. 2005;25:11489-93.

24. Eckstein M, Becker B, Scheele D, Scholz C, Preckel K, Schlaepfer TE, et al. Oxytocin facilitates the extinction of conditioned fear in humans. Biol Psychiatry. 2015;78:194-202.

25. Ferguson JN, Young LJ, Hearn EF, Matzuk MM, Insel TR, Winslow JT. Social amnesia in mice lacking the oxytocin gene. Nat Genet. 2000;25:284-8.

26. Ferguson JN, Aldag JM, Insel TR, Young LJ. Oxytocin in the medial amygdala is essential for social recognition in the mouse. J Neurosci. 2001;21:8278-85

27. Strauss GP, Keller WR, Koenig Jl, Gold JM, Ossenfort KL, Buchanan RW. Plasma oxytocin levels predict olfactory identification and negative symptoms in individuals with schizophrenia. Schizophr Res. 2015;162:57-61.

28. Woolley JD, Lam O, Chuang B, Ford JM, Mathalon DH, Vinogradov S. Oxytocin administration selectively improves olfactory detection thresholds for lyral in patients with schizophrenia. Psychoneuroendocrinology. 2015;53: 217-22.

29. Kirschbaum C, Pirke KM, Hellhammer DH. The 'Trier Social Stress Test'--a tool for investigating psychobiological stress responses in a laboratory setting. Neuropsychobiology. 1993;28:76-81.
30. Sheehan DV, Lecrubier Y, Sheehan KH, Amorim P, Janavs J, Weiller E, et al (1998). The Mini-International Neuropsychiatric Interview (M.I.N.I): the development and validation of structured diagnostic psychiatric interview for DSM-IV and ICD-10. The Journal of clinical psychiatry 59 (Suppl 20): 22-33.

31. McLaren DG, Ries ML, Xu G, Johnson SC. A generalized form of contextdependent psychophysiological interactions (gPPI): a comparison to standard approaches. Neuroimage. 2012;61:1277-86.

32. Critchley HD. Neural mechanisms of autonomic, affective, and cognitive integration. J Comp Neurol. 2005;493:154-66.

33. Johnston K, Levin HM, Koval MJ, Everling S. Top-down control-signal dynamics in anterior cingulate and prefrontal cortex neurons following task switching. Neuron. 2007;53:453-62.

34. Etkin A, Egner T, Kalisch R. Emotional processing in anterior cingulate and medial prefrontal cortex. Trends Cogn Sci. 2011;15:85-93.

35. McEwen BS, Gianaros PJ. Central role of the brain in stress and adaptation: links to socioeconomic status, health, and disease. Ann N Y Acad Sci. 2010;1186:190-22.

36. Gottfried JA, Dolan RJ. The nose smells what the eye sees: crossmodal visual facilitation of human olfactory perception. Neuron. 2003;39:375-86.

37. Wang J, Korczykowski M, Rao H, Fan Y, Pluta J, Gur RC, et al. Gender difference in neural response to psychological stress. Soc Cogn Affect Neurosci. 2007;2:227-39.

38. Stevens JS, Hamann S. Sex differences in brain activation to emotional stimuli: a meta-analysis of neuroimaging studies. Neuropsychologia. 2012;50:1578-93.

39. Cahill L, Uncapher M, Kilpatrick L, Alkire MT, Turner J. Sex-related hemispheric lateralization of amygdala function in emotionally influenced memory: an FMRI investigation. Learn Mem. 2004;11:261-6.

40. Ycaza Herrera A, Mather M. Actions and interactions of estradiol and glucocorticoids in cognition and the brain: Implications for aging women. Neurosci Biobehav Rev. 2015;55:36-52.

41. Kret ME, De Gelder B. A review on sex differences in processing emotional signals. Neuropsychologia. 2012;50:1211-21.

42. Kubzansky LD, Mendes WB, Appleton AA, Block J, Adler GK. A heartfelt response: oxytocin effects on response to social stress in men and women. Biol Psychol. 2012;90:1-9.

43. Bethlehem RAl, Lombardo MV, Lai MC, Auyeung B, Crockford SK, Deakin J, et al. Intranasal oxytocin enhances intrinsic corticostriatal functional connectivity in women. Transl Psychiatry. 2017;7:e1099.

44. Preckel K, Scheele D, Eckstein M, Maier W, Hurlemann R. The influence of oxytocin on volitional and emotional ambivalence. Soc Cogn Affect Neurosci. 2015;10:987-93.

45. Li J, Liu J, Liang J, Zhang $\mathrm{H}$, Zhao J, Huber DE, et al. A distributed neural system for top-down face processing. Neurosci Lett. 2009;451:6-10.

46. Kreuder AK, Scheele D, Wassermann L, Wollseifer M, Stoffel-Wagner B, Lee MR, et al. How the brain codes intimacy: the neurobiological substrates of romantic touch. Hum Brain Mapp. 2017;38:4525-34.

47. Blair KS, Geraci M, Smith BW, Hollon N, DeVido J, Otero M, et al. Reduced dorsal anterior cingulate cortical activity during emotional regulation and top-down attentional control in generalized social phobia, generalized anxiety disorder, and comorbid generalized social phobia/generalized anxiety disorder. Biol Psychiatry. 2012;72:476-82.

48. Bar-Haim Y, Lamy D, Pergamin L, Bakermans-Kranenburg MJ, van IMH. Threatrelated attentional bias in anxious and nonanxious individuals: a meta-analytic study. Psychol Bull. 2007;133:1-24.

49. Marsh N, Scheele D, Feinstein JS, Gerhardt H, Strang S, Maier W, et al. Oxytocinenforced norm compliance reduces xenophobic outgroup rejection. Proc Natl Acad Sci USA. 2017;114:9314-9.

50. Penn DJ, Oberzaucher E, Grammer K, Fischer G, Soini HA, Wiesler D, et al. Individual and gender fingerprints in human body odour. J R Soc Interface. 2007;4:331-40.

51. Lubke KT, Hoenen M, Pause BM. Differential processing of social chemosignals obtained from potential partners in regards to gender and sexual orientation. Behav Brain Res. 2012:228:375-87.

52. Radulescu AR, Mujica-Parodi LR. Human gender differences in the perception of conspecific alarm chemosensory cues. PLoS ONE. 2013;8:e68485. 